J. Phys. IV France 138 (2006) 89-94

(C) EDP Sciences, Les Ulis

DOI: 10.1051/jp4:2006138011

\title{
Étude spectrale d'un micro-plasma d'implosion X-pinch
}

\author{
L.E. Aranchuk ${ }^{1}$ et J. Larour ${ }^{1}$ \\ ${ }^{1}$ Laboratoire de Physique et Technologie des Plasmas (LPTP), CNRS UMR 7648, \\ École Polytechnique, 91128 Palaiseau, France
}

\begin{abstract}
Résumé. Le pincement magnétique de type X-pinch permet de réaliser un micro-plasma chaud et dense bien positionné dans l'espace qui présente une applicabilité pour la radiographie $\mathrm{X}$ d'objets peu denses de petite taille. Une batterie de diagnostics ayant tous une résolution spectrale a été installée autour d'un X-pinch alimenté par un banc de condensateurs rapide. Les résultats présentés portent sur la taille de la source intégrée en temps, sur la durée d'impulsion et sur le spectre d'émission X. Des exemples de radiographie confortent la détermination de la taille. Des spectres à haute résolution spectrale intégrés en temps (1 à $3 \mathrm{keV}$ ) montrent que les ions multichargés présents dans le plasma (Al hydrogénoïde et héliumoïde, $\mathrm{Cu}$ et Mo néonoïdes) sont les mêmes que dans les X-pinches alimentés par des générateurs pulsés bien plus puissants. Une spectroscopie large bande à haute résolution temporelle, basée sur des détecteurs innovants, a permis de mettre en évidence une émission dure sub-nanoseconde et de chiffrer la puissance spectrale instantanée et l'énergie $\mathrm{X}$ émise entre $20 \mathrm{eV}$ et $8 \mathrm{keV}$. La puissance maximale peut dépasser $1 \mathrm{GW}$ pendant $0,4-0,7 \mathrm{~ns}$. La source émet 10 à $30 \mathrm{~J}$ pendant $100-150 \mathrm{~ns}$ en dessous de $400 \mathrm{eV}$.
\end{abstract}

\section{INTRODUCTION}

Un X-pinch est composé de deux ou plusieurs fils fins, croisés entre deux électrodes de façon à se toucher en un point. Lors de l'application rapide d'une haute tension, un courant élevé circule sur chaque fil et entame la formation d'un plasma coronal. Au niveau du point de croisement, tout le courant participe à la formation d'une pression magnétique maximale qui peut alors imploser le plasma et le chauffer violemment. Ainsi, une source de rayonnement X quasi-ponctuelle peut être localisée en un endroit prédéfini et utilisée pour faire une radiographie éclair par projection.

Usuellement les X-pinches sont alimentés par des générateurs rapides composés d'un Marx et d'une ligne coaxiale à basse impédance ; la tension délivrée est alors très élevée, de 250 à $500 \mathrm{kV}$. Notre démarche a été de raisonner en terme de courant intense rapidement variable et de concevoir un générateur de courant dédié à l'explosion de fil. Le montage expérimental a déjà été décrit en détails [1]. Rappelons que l'alimentation électrique est constituée par un banc de condensateurs rapide stockant $850 \mathrm{~J}$ sous $40 \mathrm{kV}$. Un ensemble d'éclateurs à haute performance ainsi qu' une section à isolement magnétique sous vide assurent à ce système compact une inductance très faible $(16 \mathrm{nH})$ ce qui autorise une montée rapide du courant dans la charge. Cette condition est nécessaire pour arriver au pincement magnétique avant l'explosion des fils. Un courant de plus de 200kA montant en moins de 200ns a été obtenu dans un court-circuit de $6 \mathrm{nH}$. Les sections de transport à isolement magnétique nécessitent un vide secondaire, ce qui ralentit le rythme des tirs à 3 par jour.

Le rayonnement du microplasma dépendant des ions multichargés qui le forment, le matériau formant le X-pinch a été choisi parmi ceux émettant au dessus du keV, disponibles dans le commerce en fils de diamètres compatibles avec l'énergie délivrée et la possibilité mécanique du montage. Les configurations retenues sont principalement $\mathrm{Al} 20 \mu \mathrm{m}$ et $25 \mu \mathrm{m}, \mathrm{Cu} 25 \mu \mathrm{m}$, Mo $25 \mu \mathrm{m}$ et W $13 \mu \mathrm{m}$ et $18 \mu \mathrm{m}$. Le fil, lesté de plombs de 2 à $6 \mathrm{~g}$, est croisé en 2 par rotation de $180^{\circ}$ de l'anode supérieure, plus un angle destiné à rattraper le jeu mécanique du fil dans les fentes des électrodes. Pour un angle de $15^{\circ}$, un bon couplage de l'énergie se produit et on observe une émission X en montée du courant [2].

Les premières campagnes ont montré la petitesse de la taille de la source $\mathrm{X}$ et nous $\mathrm{y}$ revenons en présentant des applications radiographiques qui la confirment. On avait aussi mesuré une durée 
d'émission du rayonnement dur ( $>1,5 \mathrm{keV})$ assez brève, environ 1,5ns, présentant des indices de pics multiples [2]. Cependant la littérature rapporte des séries de 2 ou 3 impulsions plus brèves d'un facteur $10[3,4]$. Il était nécessaire de clarifier si cette différence venait du type d'alimentation électrique du $\mathrm{X}$-pinch ou de la réponse du détecteur. Cela a été possible avec des détecteurs à la fois très sensibles et très rapides fournis par l'Institut de recherche sur les phénomènes impulsionnels de Moscou. Outre cette étude temporelle, ces détecteurs ont été utilisés comme capteurs calibrés afin de mesurer l'énergie $\mathrm{X}$ émise et la puissance spectrale de façon absolue.

\section{TAILLE DE SOURCE ET APPLICATION RADIOGRAPHIQUE}

On avait rapporté des mesures préliminaires de taille de la source $\mathrm{X}$ inférieures à $100 \mu \mathrm{m}(\mathrm{E}>1,5 \mathrm{keV})$ par sténopé de diamètre $10 \mu \mathrm{m}$ [2]. Ensuite, par analyse de pénombre en projection d'un trou de $200 \mu \mathrm{m}$ et d'une grille de fil $15 \mu \mathrm{m}[5,6]$ on a pu dépasser la limitation du sténopé et mesurer des tailles de 8 à $14 \mu \mathrm{m}$ pour un rayonnement d'énergie supérieure à $2 \mathrm{keV}$ (filtration Ti $8 \mu \mathrm{m}$ ).

La source étant ainsi définie, elle est convenable pour radiographier des objets de petite taille et peu absorbants. On a choisi la radiographie de projection avec un grandissement de 4,2 et une détection sur film DEF, ensuite numérisé sur 16 bits à 1200 ppi. Un premier essai a été fait avec une graine de pissenlit (Fig. 1) formée de fibres très fines rayonnant autour d'un pied central. La radiographie avec une quasi-monosource (X-pinch de $\mathrm{Al} 25 \mu \mathrm{m}, \mathrm{E} \approx 1,6 \mathrm{keV}$, photo a) montre une bonne restitution de toutes le fibres, quelle que soit leur orientation, avec une perception de la diminution d'épaisseur en bout de fibre. Au contraire, une source étendue le long de l'axe vertical anode-cathode (X-pinch de Mo $25 \mu \mathrm{m}, \mathrm{E} \approx 2,6 \mathrm{keV}$, photo $\mathrm{b}$ ) montre un dédoublement des images des fibres perpendiculaires à cette direction. Des fils de $\mathrm{W}$ de 25 et $50 \mu \mathrm{m}$, ajoutés à titre de témoin, sont résolus avec les mêmes limitations. Le second test a été fait avec une carcasse de mouche desséchée, c'est à dire un ensemble de tiges et de parois en kératine, polymère naturel riche en $\mathrm{C}, \mathrm{N}$ et $\mathrm{S}$. Malgré la légèreté des atomes, un très bon contraste est obtenu avec un X-pinch de W $13 \mu \mathrm{m}$, à la fois sur les structures internes et sur les barbes externes (Fig. 1). Un zoom sur l'oeil permet de distinguer le réseau des facettes, ce qui confirme une taille de source $\mathrm{X}$ de $10 \mu \mathrm{m}$ pour résoudre ce type de détail.

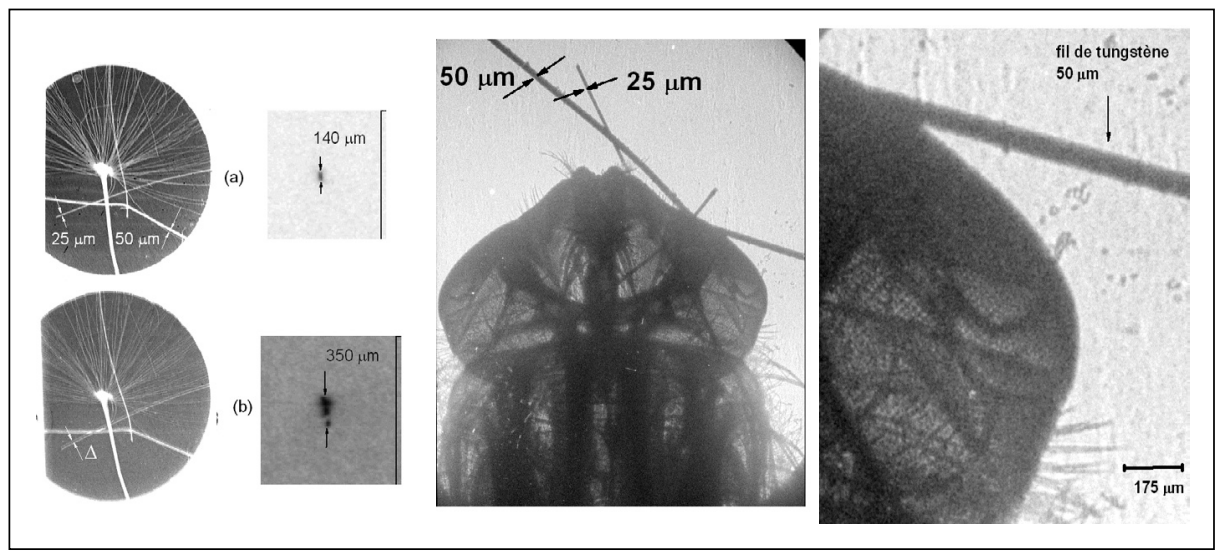

Figure 1. Radiographies par X-pinch d'une graine de pissenlit avec une monosource Al $25 \mu \mathrm{m} \mathrm{E} \approx 1,6 \mathrm{keV}$ (a) et avec une source étendue Mo $25 \mu \mathrm{m} \mathrm{E} \approx 2,6 \mathrm{keV}$ (b). A droite, radiographie d'une tête de mouche domestique desséchée et détail de l'oeil droit. Des fils de tungstène donnent l'échelle. Dans les deux cas, le grandissement est 4,2 . 


\section{SPECTRE D'ÉMISSION X}

\subsection{Spectroscopie à haute résolution intégrée en temps}

La première approche a été d'exploiter la petitesse de la source et de caractériser l'émission du point chaud décrit plus haut. En effet, pour un spectrographe à cristal plan ou courbe, les raies ne seront pas notablement élargies par notre source X. Des spectres ont été enregistrés (Fig. 2) avec des cristaux plans KAP, ADP et PET, et confirmés autant que possible avec un spectrographe à mica convexe $(\mathrm{R}=10,5 \mathrm{~mm})$ travaillant aux ordres 1 et 2 . Un filtrage Be $15 \mu \mathrm{m}$ associé à une détection par film DEF nous donne une sensibilité utile au dessus de $600 \mathrm{eV}$ (en dessous de 20Ã). Un filtrage supplémentaire par $3 \mu \mathrm{m}$ d'Aluminium permet de vérifier l'ordre par le taux d'absorption. Il est à noter que les spectres des ions multichargés sont qualitativement les mêmes que ceux observés sur des X-pinches puissants $[3,4]$. La principale différence porte sur les largeurs des raies, plus fines dans notre cas, ce qui conduit à conclure que notre plasma est moins dense mais formé des mêmes ions. Cette étude sera poursuivie avec un cristal KAP convexe pour comparer à des codes sur une large gamme spectrale.
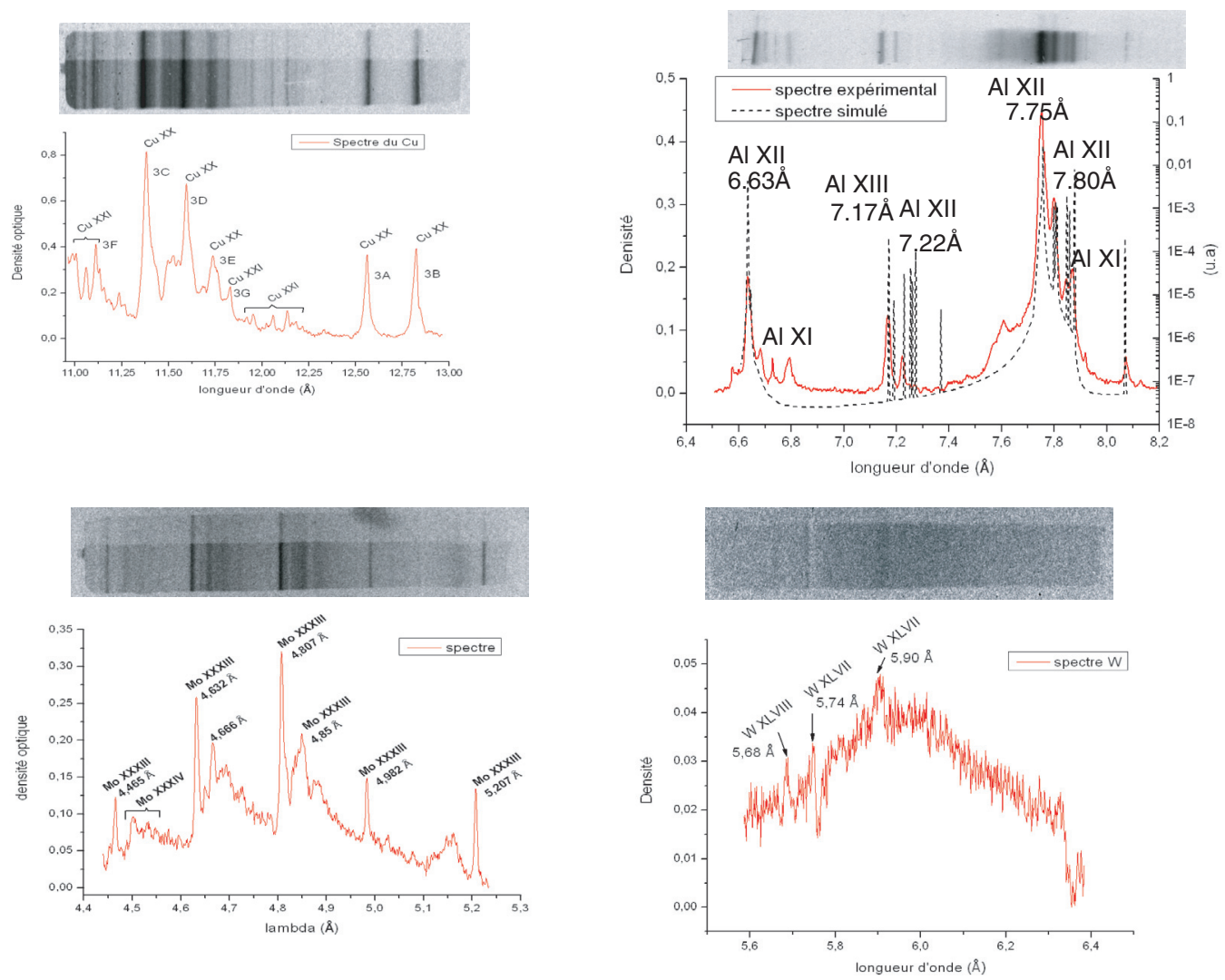

Figure 2. Spectres intégrés en temps et profils numérisés de densité optique. La partie supérieure du spectre correspond à une atténuation supplémentaire de $\mathrm{Al} 3 \mu \mathrm{m}$, au dessus de $\mathrm{Be} 15 \mu \mathrm{m}$. L'étalonnage en longueur d'onde est fait par la géométrie puis rapproché des raies de la littérature. De gauche à droite et de haut en bas : spectre des ions cuivre riche en $\mathrm{CuXX}$ et $\mathrm{CuXXI}$, spectre d'Al hydrogénoïde et héliumoïde confronté à une modélisation FLY $\left(250 \mathrm{eV}, 10^{21} \mathrm{e} / \mathrm{cm}^{3}\right)$, spectre de Mo riche en ions MoXXII et MoXXXIII, spectre de W avec une suspicion d'ion nickelloïde WXLVII. 


\subsection{Spectroscopie large bande résolue en temps}

Cette étude est menée avec des détecteurs rapides irradiés simultanément. Le jeu de détecteurs est constitué de 7 diodes à vide DPX de temps de montée $\tau<200$ ps et de 4 diodes $\mathrm{p}$-i-n $(\tau<1,5 \mathrm{~ns})$. L'enregistrement des transitoires est fait entre 2 et 20Géch/s sur des oscilloscopes LeCroy de bande passante 1 à $6 \mathrm{GHz}$. On a vérifié l'isotropie du rayonnement en interchangeant les détecteurs dans un plan médian entre anode et cathode. On installe radialement une série de $\mathrm{i}=6$ à 11 détecteurs rapides très sensibles à des distances adaptées, entre $0,3 \mathrm{~m}$ et $2,4 \mathrm{~m}$. Les diodes photoémissives $\mathrm{X}$ sont filtrées par grille et écran (tableau 1) les diodes p-i-n travaillent en détection intégrale derrière grille et écran.

La première étude porte sur la durée d'impulsion. La conjonction de détecteurs très sensibles et d'une forte tension de polarisation (400V) avec des tés HT très rapides (Picosecond Pulse Labs 5531, $10 \mathrm{GHz}$ ) permet l'observation d'impulsions subnanosecondes. La figure 3 montre la variation de forme et de largeur du pic de rayonnement $\mathrm{X}$ en fonction de la sensibilité spectrale. Comme dans les systèmes de haute puissance électrique [3, 4], nous enregistrons une émission sub nanoseconde sur la partir dure du rayonnement d'un X-pinch Mo $25 \mu \mathrm{m}$, c'est à dire le spectre de raies vers 2,6keV. Ceci confirme la moindre dépendance du fonctionnement du X-pinch avec le type de banc d'énergie, dans la mesure où un niveau de courant suffisant est atteint avant la destruction des fils.

Le deuxième objectif est d'estimer la puissance spectrale de la source $\mathrm{X}$ et l'énergie contenue dans l'impulsion. Pour cela, nous utilisons les signaux transitoires délivrés par les capteurs décrits plus haut. La procédure d'exploitation spectrale des signaux est la suivante. On enregistre les $i$ canaux et on dispose ainsi d'un jeu de réponses expérimentales $R_{i}(t)$ pour des sensibilités de détection $S_{i}(E)$ fournies par le fabricant, corrigées de la géométrie et des absorbeurs sur une gamme étendue $20 \mathrm{eV}<E<10 \mathrm{keV}$. Ces réponses sont exploitées, soit en valeur instantanée $(\delta t=1 \mathrm{~ns})$ autour du pic (Figure $4 \mathrm{a})$, soit intégrées sur les phases principales de l'émission (intervalle de temps de 1ns à 200ns). On applique une méthode de gradient à recherche aléatoire de la pente [7] pour trouver, par itération, le meilleur spectre $P(E, t)$, défini par paliers sur $j$ intervalles $(i \geq j)$ et reproduisant aux moindres carrés les $i$ réponses expérimentales : $R_{i}(t) \approx \int P(E, t) . S_{i}(E) . d E$. La robustesse de l'algorithme a été testée sur

Tableau 1. Caractéristiques des détecteurs DPX rapides.

\begin{tabular}{|c|c|c|c|c|c|c|c|}
\hline cathode diam. Mm & $\mathrm{Al} \emptyset 6$ & $\mathrm{Al} \emptyset 6$ & $\mathrm{Au} \emptyset 6$ & $\mathrm{Au} \emptyset 61$ & $\mathrm{Au} \emptyset 6$ & $\mathrm{Au} \emptyset 6$ & $\mathrm{Au} \emptyset 16$ \\
\hline filtre épaisseur mm & $\mathrm{Al} 0,4$ & $\mathrm{Zr} 0,2$ & $\mathrm{Ag} 0,15$ & Polypropylène 4 & $\mathrm{Ti} 0,25$ & $\mathrm{Cu} 0,15$ & $\mathrm{Be} 25$ \\
\hline Désignation & $\mathrm{N} 6$ & $\mathrm{~N} 5$ & $\mathrm{~N} 4$ & $\mathrm{~N} 3$ & $\mathrm{~N} 2$ & $\mathrm{~N} 1$ & $\mathrm{XRD} 16$ \\
\hline gamme sensibilité eV & $20-60$ & $60-200$ & $115-405$ & $205-290$ & $220-465$ & $325-935$ & $>950$ \\
\hline $\begin{array}{c}\text { sensibilité maxi } \\
\mu \mathrm{A} * \mathrm{~cm}^{2} / \mathrm{W}\end{array}$ & 350 & 50 & 20 & 30 & 26 & 16 & 60 \\
\hline
\end{tabular}

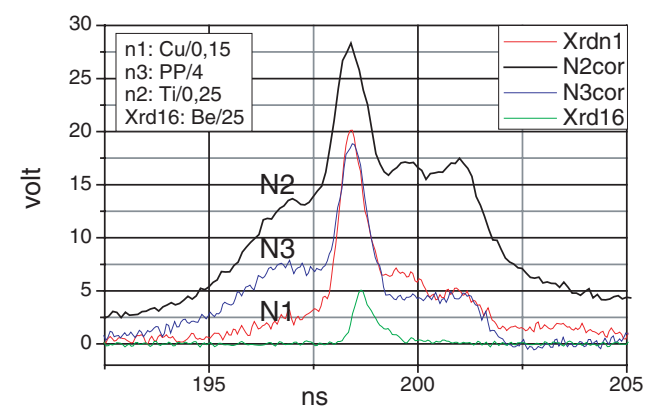

Figure 3. Signaux des détecteurs de rayonnement montrant la structure du pic pour un X-pinch Mo $25 \mu \mathrm{m}$. On remarque le niveau élevé des signaux DPX ( 5 à $28 \mathrm{~V}$ au pic), bien au dessus du bruit $(0,1 \mathrm{~V})$ qui permet l'exploitation sur une large dynamique. Les noms des détecteurs renvoient au tableau 1. 


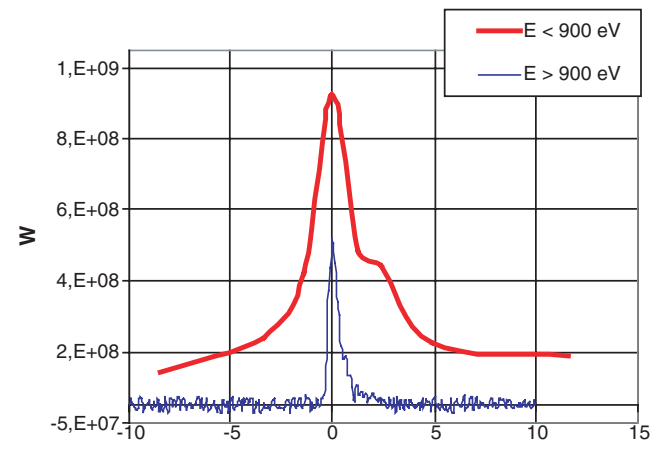

ns



b)

Figure 4. Reconstitution spectrale dans $4 \pi$ pour un X-pinch Mo $25 \mu \mathrm{m}$ : a) transitoire de puissance autour du pic d'émission $\mathrm{X}$ en dessous et au dessus de $900 \mathrm{eV}, \mathrm{b}$ ) puissance spectrale à l'instant du pic reconstituée à partir des DPX pour la partie à basse énergie $(<935 \mathrm{eV})$ et à partir des diodes p-i-n pour la partie à haute énergie $(>650 \mathrm{eV})$.

des spectres synthétiques (Planck + raies) vis à vis du nombre et des bornes des paliers et du pas d'intégration ( $\left.\delta E=E_{\max } / 1024\right)$. Pour une bonne restitution, la distribution en énergie des sensibilités des détecteurs doit couvrir tout l'intervalle et les recouvrements doivent rester peu importants. C'est le cas pour les filtrations rappelées au tableau 1. Un exemple d'application est donné Figure $4 \mathrm{~b}$ où l'on a reconstitué le spectre émis à l'instant du pic. La partie basse énergie, de 20 à $935 \mathrm{eV}$, est déduite des réponses des $7 \mathrm{DPX}$ et la partie haute énergie $(650-3000 \mathrm{eV})$ des réponses des $p-i-n$. L'écart dans la zone de recouvrement permet d'évaluer la précision de la méthode.

La méthode a été employée pour analyser les grandes phases de développement du X-pinch dans les différentes configurations : élément, nombre de pics, nombre de points source. La comparaison est en cours avec les spectres de raies expérimentaux (cf \$3.1). Sur un tir typique Mo $25 \mu \mathrm{m}$ à monosource, on peut isoler les éléments suivants. Avant le pic, la puissance rayonnée - en dessous de 400eV - varie de 50 à 100MW lors d'une phase de préparation durant environ 110-140ns. Dans les 3-5ns avant le pic, on observe un chauffage du plasma et une montée de rayonnement dans la gamme $200-400 \mathrm{eV}$ mais pas au dessus, pour une puissance de 200-400MW. Ensuite, le pic de puissance peut dépasser $1 \mathrm{GW}$ dont 20 à $35 \%$ est émis au dessus de $900 \mathrm{eV}$ et la durée pour cette énergie va de 400 à 700 ps. La phase de refroidissement dure 75 à 100ns au niveau de 100 à 400MW pour une énergie XUV inférieure à $400 \mathrm{eV}$. Au final, la source X-pinch émet de 10 à 30J pendant 100 - 150ns dans la gamme spectrale $10-400 \mathrm{eV}$.

\subsection{Spectroscopie d'absorption}

La microsource $X$ est également capable de réaliser une spectroscopie d'absorption de films minces prévus pour filtrer les DPX. Le dispositif de spectroscopie à cristal plan est modifié en remplaçant le filtre supplémentaire d'aluminium par le filtre inconnu. On dispose donc en un tir d'un spectre de premier ordre filtré par Be $15 \mu \mathrm{m}$, avec ou sans absorbeur. Le film Kodak DEF est numérisé à 1200ppi et les densités optiques sont mesurées à énergie fixe sur la largeur d'une raie ou sur un groupe de raies.

Les mesures, qui s'étendent de 1000 à $3000 \mathrm{eV}$, ont mis en évidence les fronts K des deux dopants (P à $2140 \mathrm{eV}$ et $\mathrm{Cl}$ à $2820 \mathrm{eV}$ ) et les tendances (Fig. 5). Une procédure d'ajustement permet de trouver la composition la plus probable en masses surfaciques de carbone et du dopant. Il est à signaler que dans le cas du phosphore, une granulométrie importante du dopant fait apparaître des taches opaques submillimétriques qui sont prises en compte pour moyenner sur une aire d'intégration suffisante. 




Figure 5. Transmission de films polymères approchée par des compositions $\mathrm{C}_{\mathrm{x}} \mathrm{P}_{\mathrm{y}}$ et $\mathrm{C}_{\mathrm{z}} \mathrm{Cl}_{\mathrm{t}}$. Les fronts d'absorption de $\mathrm{P}$ à $2140 \mathrm{eV}$ et $\mathrm{Cl}$ à $2820 \mathrm{eV}$ sont accessibles au X-pinches.

\section{CONCLUSION}

Le X-pinch est une source X qui reste ponctuelle et brève, alimentée par un banc de consensateurs rapide et compact délivrant 200kA en 200ns. Comme les systèmes de grande taille, il donne un plasma d'ions fortement chargés, même pour des matériaux de Z élevé, si la masse de la cible reste adaptée à l'énergie stockée. La taille de la source descend à $10 \mu \mathrm{m}$ au dessus de $2 \mathrm{keV}$ ce qui permet des radio-graphies en projection. Le spectre d'émission est caractérisé par les raies ioniques, $\mathrm{K}$ pour $\mathrm{Al}, \mathrm{L}$ pour $\mathrm{Mo}$ et $\mathrm{Cu}$, M pour W. Des détecteurs rapides ont permis de chiffrer la puissance spectrale et l'énergie X absolues. L'émission totale culmine au dessus de $1 \mathrm{GW}$ pour une énergie d'une dizaine de joules.

\section{Remerciements}

Les auteurs remercient l'Institut de recherche sur les phénomènes impulsionnels de Moscou pour la fourniture des détecteurs et M.I. Ivanov pour sa contribution au développement des détecteurs et la préparation de l'expérience.

\section{Références}

[1] Aranchuk L.E., Chuvatin A. and Larour J., Review Sci. Instrum. 75 (2004) 69-74.

[2] Aranchuk L.E., Larour J. and Chuvatin A., IEEE Trans. on Plasma Sci. 33 (2005) 990-996.

[3] Hansen S.B., Shlyaptseva A.S., Pikuz S.A., Shelkovenko T.A., Sinars D.B., Chandler K.M. and Hammer D.A., Phys. Rev. E 70 (2004) 026402.

[4] Sinars D.B., Pikuz S.A., Shelkovenko T.A., Chandler K.M., Hammer D.A. and Apruzese J.P., J. Quant. Spectrosc. Radiat. Transfer 78 (2003) pp. 61-83.

[5] Aranchuk L.E. and Larour J., "Submicrosecond X-pinch as a source of point-like radiation and multi-charged hot plasma”, BEAMS 2004, St Pétersburg, Russie, G. Mesyats, V. Smirnov, V. Engelko Eds (Efremov Inst., St Petersburg, Russie, 2005), pp. 750-753.

[6] Aranchuk L.E. et Larour J., "Source quasi-ponctuelle par X-pinch en régime sub- $\mu$ s”, UVX, SaintEtienne, 2004, Y. Ouerdane et P. Laporte Eds (EDP Sciences, Les Ulis, France, 2005) pp. 21-24.

[7] Aranchuk L.E., Larour J. Buryakov V.L., Ivanov M.I. and Alexandrin S.U., "Time resolved spectra of X-ray radiation from a submicrosecond X-pinch plasma”, Dense Z-pinch Conf., Oxford, G.B., 2005, J. Chittenden Ed (AIP Conf. Proc. 808, New-York, 2006), pp. 117-120. 